研究

\title{
Fiber Bragg Grating Hydrophone Array using Feedback Control Circuit: Time-division Multiplexed and Thermally Stabilized Operation
}

\author{
Satoshi TANAKA, Hiroki YOKOSUKA and Nobuaki TAKAHASHI \\ (Department of Communications Engineering, National Defense Academy) \\ satoshi@nda.ac.jp
}

(Received 8 August 2005)

\begin{abstract}
:
A novel fiber Bragg grating (FBG) hydrophone array has been proposed in order to realize multipoint underwater sound detection with thermal stabilization. The interrogation technique of the FBG hydrophone to be arrayed is based on an intensity-modulation scheme, in which a tunable laser is used for an optical source with its oscillation wavelength tuned to a slope of the reflection spectrum curve of the FBG. The partially reflected light from the FBG is modulated by an acoustic wave and offers a sensor output. For fabricating the FBG hydrophone array, an optical switch and a specially designed feedback circuit have been employed. The arrayed FBG hydrophones are connected in parallel with the output ports of the optical switch so that the time-division multiplexed (TDM) operation is performed by selecting the optical paths to the hydrophones. In the TDM operation, the feedback circuit enables us to control the oscillation wavelength automatically in accordance with the reflection spectrum for the selected FBG hydrophone. In addition, the automatic wavelength control allows the stabilization of the sensor output against temperature variations. In the experiment, two-point TDM sound detection with a thermally stabilized operation has been successfully demonstrated.
\end{abstract}

Classification: Miscellaneous (Observations, Measurements etc.)

Keyword: Hydrophone, Acoustic sensor, Fiber optic sensor, Underwater sound detection, Fiber Bragg grating, Time-division multiplexing

\section{Introduction}

Recently, fiber Bragg gratings ${ }^{1,2)}$ (FBGs) have attracted much attention as sensing elements for physical parameters such as mechanical deformation, hydrostatic pressure, elastic vibration, acoustic wave, and temperature. This is because FBG sensors ${ }^{3-5)}$ have fol- lowing advantages in addition to that of conventional fiber optic sensors: small dimensions, simple structure, and ease in multiplexing sensors. In most FBG sensors, their principles of operation are based on shift in Bragg wavelength of the FBG or in FBG spectrum curve when the FBG is subjected to the measurand that can 
be converted into the physical parameters. The interrogation schemes of such sensors are fundamental techniques and mainly divided into two types: one is based on direct measurement of FBG spectrum curve, and the other is based on intensity modulation converting the wavelength shift to the optical intensity. Since the latter type needs no spectroscopic detection system, it is a very attractive approach for the FBG sensor performed in real time, especially for vibration of solid ${ }^{6-8)}$ or acoustic wave in water. ${ }^{9-11)}$ In this type of sensors, a narrow-bandwidth optical source (e.g. laser) is utilized and its wavelength is tuned to the slope of the FBG spectrum curve, i.e., operation point. As a result, the output light partially reflected (or transmitted) at the FBG is modulated in intensity when the acoustic wave is applied to the FBG. We have previously reported FBG hydrophones based on the intensity-modulation scheme, in which real time observation of the waveform and spectrum of the underwater sound is demonstrated.9 ${ }^{90}$ For a practical use of such sensors, however, the multiplexed sensing operation is required, in that the multiple point underwater sound detection can be realized using a single sensor system. Additionally, thermally stabilized operation in the sound detection is also desirable, since the FBG sensor is likely to suffer from environmental temperature variation ${ }^{12)}$. In the preceding paper we have reported a temperature stabilized FBG sensor array for a vibration of solid by using paired FBGs, ${ }^{13)}$ in which one of the FBGs is embedded in the solid sample and subjected to both the vibration and temperature variation, but the other is touched loosely with the sample to be affected by only the temperature so that it can be used for a temperature compensation. This technique, however, is difficult to apply to the FBG hydrophone. This is because the effects of temperature and acoustic wave on the FBG could not be separated from each other in water. From these points as background, in this paper a novel time-division multiplexed (TDM) FBG hydrophone array is pro- posed, in which multiple underwater sound detections and immunity to environmental temperature variations can be achieved. The key technique of the hydrophone array is a feedback circuit to control a wavelength of a tunable laser. The feedback control enables us to adjust the wavelength of the tunable laser to the operation point of the FBG automatically. The automatic wavelength control allows the compensation of the change of the operation point in the TDM operation and in the environmental temperature variation as well. In the experiment, by constructing the FBG hydrophone array in which two FBG sensors are arranged in parallel, TDM operation with thermal stabilization is demonstrated.

\section{Principle of Operation}

\subsection{FBG Hydrophone Array}

The FBG hydrophone designed here is operated in a reflection mode: with reference to Fig. 1, the wavelength of a laser source is tuned to a slope of FBG reflection spectrum curve, namely, $R(\lambda)$. Since an acoustic wave induces the periodical shift of the spectrum curve, $R(\lambda)$, as well as Bragg wavelength, $\lambda_{\mathrm{B}}$, in a wavelength domain, the partially reflected light of the laser is modulated in intensity and offers a sensor output. Let $I_{\text {in }}$ and $\lambda_{\text {in }}$ be an intensity and a wavelength of the incident light from the laser source, respectively, and the intensity of the reflected light $I_{\text {out }}$ can be written as,, 911$)$

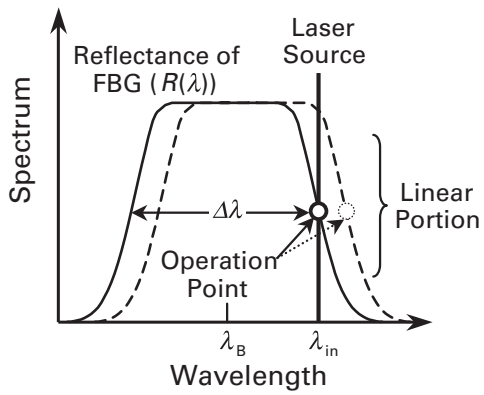

Fig. 1 Schematic view of reflection spectrum of FBG, $R(\lambda)$, and oscillation spectrum of laser source. 


$$
I_{\text {out }}=I_{\text {in }} R\left(\lambda_{\text {in }}\right)+\left.I_{\text {in }} \frac{\partial R(\lambda)}{\partial \lambda}\right|_{\lambda \approx \lambda_{\text {in }}} \eta p \sin 2 \pi f t
$$

where $R\left(\lambda_{\text {in }}\right)$ is the reflectance of the FBG at $\lambda_{\text {in }}, p$ and $f$ : the amplitude and frequency of the sound pressure due to the acoustic wave, respectively, $\eta$ : the rate of the spectrum-shift to the sound pressure, and $\partial R(\lambda) / \partial \lambda \mid \lambda \approx \lambda_{\text {in }}$ : the gradient of $R(\lambda)$ around $\lambda_{\text {in }}$. Here, $\partial R(\lambda) /\left.\partial \lambda\right|_{\lambda \approx \lambda_{\text {in }}}$ is almost constant under the assumption that $\lambda_{\text {in }}$ is adjusted to the linear position of $R(\lambda)$ (operation point) and the shift of $R(\lambda)$ induced by the acoustic wave is much smaller than the reflection bandwidth of the FBG. As a result, the sound pressure can be observed directly from the ac component of $I_{\text {out }}$, i.e., the second term of Eq. (1). It is noted here that $\partial R(\lambda) / \partial \lambda \mid \lambda \approx \lambda_{\text {in }}$ determines the sensitivity of the sensor output from the FBG hydrophone.

To fabricate a FBG hydrophone array an optical switch is used for a TDM operation as shown in Fig. 2: two FBG hydrophones are connected to the output ports of the optical switch and the optical paths to the respective hydrophones (sensor 1 and sensor 2) are switched alternately. The TDM sensor output from the arrayed hydrophones is thus obtained at the optical receiver (OR). In this scheme, the TDM operation could be realized only by switching the optical paths if the FBGs having the identical reflection spectra are used for the hydrophones. However, in general, spectral properties of FBGs (e.g. $\lambda_{\mathrm{B}}$ and $\Delta \lambda$ ) are different from each other as indicated by solid and dashed line in Fig. 1, even if the FBGs are fabricated in the same process. In this case, the operation points on the FBG spectra become different from each other. Therefore it is necessary to adjust the wavelength of the laser source in accordance with the operation point for the different FBG so that it may not deviate from the linear portion of $R(\lambda)$. In addition to the TDM operation, the adjustment of the wavelength is desirable when the temperature is considerably varied. This is because the reflection spectrum curve shifts toward longer or shorter wavelength whether the FBG temperature increases or decreases, so that the operation point depends on the temperature. Accordingly, automatic compensation of the operation point is an important technique for not only fabricating a FBG hydrophone array but also the temperature stabilization.

\subsection{Feedback Control Circuit}

In order to compensate the operation point, a feedback circuit has been devised, which control the oscillation wavelength of the DFB laser source to keep the relative position of the oscillation wavelength to the FBG reflection spectrum. The block diagram of the feedback control circuit is shown in Fig. 3. The output light from the FBG hydrophone is converted into a voltage signal $V_{\text {out }}$ at the optical receiver (OR) as follows:

$$
V_{\text {out }}=\alpha I_{\text {out }}=V_{\text {dc }}+V_{\text {ac }} \text {, }
$$

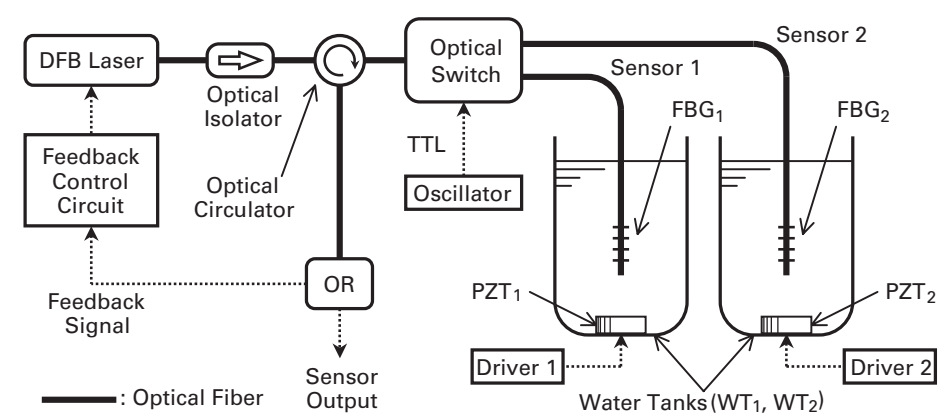

Fig. 2 Experimental setup. 


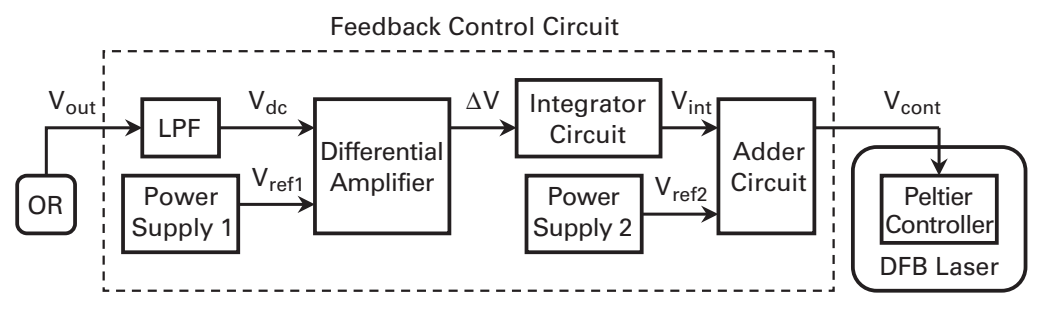

Fig. 3 Block diagram of feedback control circuit.

where, $\alpha$ is the optical/electrical conversion efficiency of OR. Substituting Eq. (1) to Eq. (2), the dc and ac components of $V_{\text {out }}, V_{\mathrm{dc}}$ and $V_{\mathrm{ac}}$, are given, respectively, by

$$
V_{\mathrm{dc}}=\alpha I_{\text {in }} R\left(\lambda_{\text {in }}\right)
$$

and

$$
V_{\mathrm{ac}}=\left.\alpha I_{\mathrm{in}} \frac{\partial R(\lambda)}{\partial \lambda}\right|_{\lambda \approx \lambda_{\text {in }}} \eta p \sin 2 \pi f t
$$

As can be seen in Eq. (3), $V_{\mathrm{dc}}$ corresponds to the operation point. With reference to Fig. $1, V_{\mathrm{dc}}$ increases or decreases when $R(\lambda)$ changes into longer or shorter wavelength. Therefore the compensation of the operation point can be achieved by adjusting the wavelength of the laser so as to keep $V_{\mathrm{dc}}$ constant. In the feedback circuit $V_{\text {out }}$ from OR is fed into a low pass filter (LPF) to extract $V_{\mathrm{dc}}$. Then, $V_{\mathrm{dc}}$ and the output voltage from the reference power supply $1, V_{\text {refl }}$, are compared and amplified at the differential amplifier, and their difference, $\Delta V$, is integrated by the integrator circuit. The sum of the output voltage from the integrator circuit, $V_{\text {int }}$, and the output voltage from the reference power supply $2, V_{\text {ref2 }}$, yields the feedback signal, $V_{\text {cont }}$, and is applied to a built-in Peltier element of a DFB laser module. The Peltier control enables the temperature tuning of the laser electrically. In this configuration, the feedback operation can be proceeded as follows. Before the feedback operation, the integrator circuit is turned off, i.e., $V_{\text {int }}=0$, and the oscillation wavelength of the laser is tuned to the operation point of one of the FBGs by adjusting only $V_{\text {cont }}$. At the beginning of the operation, $V_{\text {refl }}$ is adjusted to be equal to $V_{\text {dc }}$ so that $\Delta V$ should be zero, and then the integrator circuit is turned on. When the operation point is moved due to the exchange of the switched FBG in the TDM operation, as mentioned above, $V_{\mathrm{dc}}$ varies and $\Delta V$ comes off from zero. In this case, $\Delta V$ is integrated and added to $V_{\text {ref2 }}$ so that $V_{\text {cont }}$ changes, which results in the shift of the oscillation wavelength of the laser. The wavelengthshift continues until $\Delta V$ becomes zero. Therefore $V_{\mathrm{dc}}$ is return to the initial value of $V_{\mathrm{dc}}$, namely, $V_{\text {ref1 }}$. Consequently, the relative position of the oscillation wavelength to the spectrum curve can be kept constant by use of the feedback control circuit. Similarly, this feedback control is effective to the spectral shift of the FBG induced by temperature variation.

\section{Experiment}

Figure 2 shows the experimental setup of the FBG hydrophone array for a demonstration. A distributed feedback (DFB) semiconductor laser is used as an optical source of which oscillation wavelength can be tuned from 1555 to $1560 \mathrm{~nm}$ by adjusting a voltage to the Peltier controller. This laser is driven in an automatic power control (APC) mode so that the output power of the laser can be kept constant even in a wavelength tuning. The output light from the laser source passes through an optical isolator and an optical circulator, and is coupled into an input port of a two-channel optical switch. As shown in the figure, two FBG sen- 
sors are connected in parallel to the output ports of the optical switch so that the TDM operation is carried out by switching the optical path toward $\mathrm{FBG}_{1}$ or $\mathrm{FBG}_{2}$. Both the FBGs are immersed into water and work as FBG hydrophones (sensor 1 and sensor 2). As mentioned in the previous section, the oscillation wavelength of the laser is tuned to the operation point for $\mathrm{FBG}_{1}$ or $\mathrm{FBG}_{2}$, at which the partially reflected light is modulated in intensity if the acoustic wave is applied. The resultant reflected light is coupled into the optical circulator again to impinge on the optical receiver (OR). The output signal from OR offers sensor output and is used for the feedback control.

To fabricate a FBG hydrophone array, we used a pair of FBGs that were designed to have similar spectral parameters, i.e., Bragg wavelengths and bandwidth. The reflection spectra of the FBGs measured at a room temperature of $28{ }^{\circ} \mathrm{C}$ are shown in Fig. 4 together with a typical oscillation spectrum of the DFB laser: the solid and dashed curves are for $\mathrm{FBG}_{1}$ and $\mathrm{FBG}_{2}$ respectively, and the dotted curve for the laser. The spectral parameters of $\mathrm{FBG}_{1}$ and $\mathrm{FBG}_{2}$ were, respectively, estimated to be as follows: $1556.80 \mathrm{~nm}$ and $1556.85 \mathrm{~nm}$ for the Bragg wavelength, and 1.30 $\mathrm{nm}$ and $1.31 \mathrm{~nm}$ for the bandwidth. For the demonstration of the two-point TDM underwater sound detections, both the FBGs were immersed into the water tanks, $\mathrm{WT}_{1}$ and $\mathrm{WT}_{2}$, respectively (refer to Fig. 2). Then acoustic waves were applied to the FBGs simul-

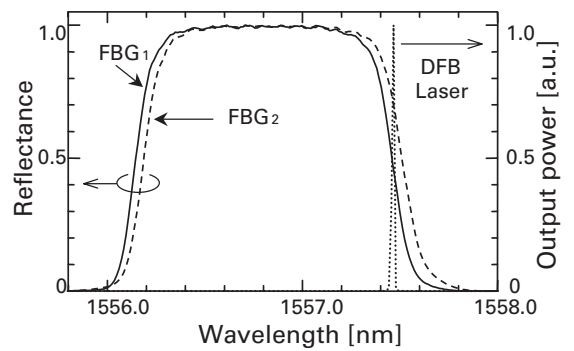

Fig. 4 Measured reflection spectra of $\mathrm{FBG}_{1}$ (solid curve) and $\mathrm{FBG}_{2}$, (dashed curve) and oscillation spectrum of DFB laser (dotted curve). taneously using piezoelectric transducers $\left(\mathrm{PZT}_{1}\right.$ and $\mathrm{PZT}_{2}$ ) driven by sinusoidal voltage signals at their respective resonant frequencies of 16.5 and $16.6 \mathrm{kHz}$. By changing the output port of the optical switch periodically using a transister-transister logic (TTL) signal, the TDM sensor output was obtained from OR.

\section{Results and Discussions}

To confirm the performance of the feedback control circuit, the optical switch was operated with a switching interval of $25 \mathrm{~s}$, and only the dc component of the sensor output $V_{\mathrm{dc}}$ was monitored without the application of the acoustic waves. An example of the observed output is shown in Fig. 5: (1) and (2) denote the output from the sensor 1 and sensor 2, respectively, and (a) and (b) indicate the output before and after the feedback control, respectively. As can be seen from the figure, the output takes two kinds of level without the feedback control. When the feedback circuit is turned off, the oscillation wavelength of the laser source is fixed so that the output of sensor 2 is different from that of sensor 1. On the other hand, with the feedback control the output level is kept constant in the TDM operation except during the transient disturbances at the switching. This means that the compensation of the operation point is achieved by use of the feedback con-

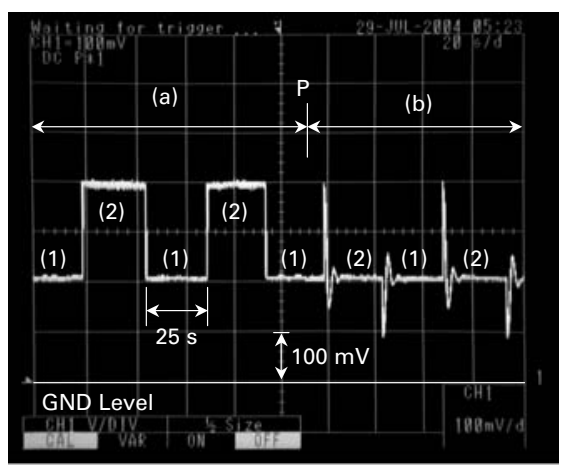

Fig. 5 Monitored dc component of sensor output without (a) and with (b) feedback control: output from sensor 1 and sensor 2 is denoted by (1) and (2), respectively. Feedback control circuit is turned on at a point $\mathrm{P}$. 
trol circuit. Typical waveforms of the sensor outputs are shown in Figs. 6 (a) and 6 (b). As mentioned above, $\mathrm{PZT}_{1}$ and $\mathrm{PZT}_{2}$ were driven in a sinusoidal mode and their driving frequencies were 16.5 and 16.6 $\mathrm{kHz}$, respectively. Since the distortions of the waveforms are considerably small as shown in the figures, it is expected that the FBG hydrophones exhibit linear responses. Figure 7 shows the output of the sensor 1 as a function of sound pressure level. As can be seen from the figure, the output from the hydrophone is in proportion to the applied sound pressure.

To examine the thermally stabilized operation of the underwater sound detection, the magnitude of the sensor output from sensor 1 was monitored while the sound pressure level around the sensor and the frequency of $\mathrm{PZT}_{1}$ were kept constant but the water temperature of $\mathrm{WT}_{1}$ was varied from 30 to $80{ }^{\circ} \mathrm{C}$. The obtained result is shown in Fig. 8. The plots with open square show the sensor outputs when the feedback circuit was turned off, i.e., the sensor was not thermally stabilized. The plots with solid circle show the sensor output when the feedback circuit was turned on, i.e., the sensor was operated with the thermal stabilization.
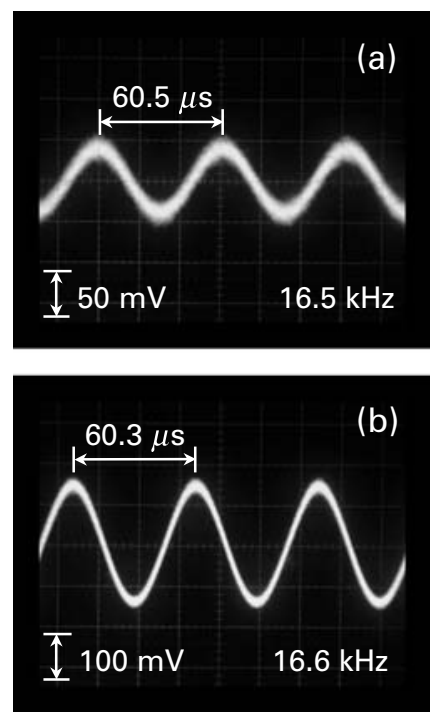

Fig. 6 Observed output waveforms obtained from sensor 1 (a) and sensor 2 (b).



Fig. 7 Sensor output as a function of sound pressure level.

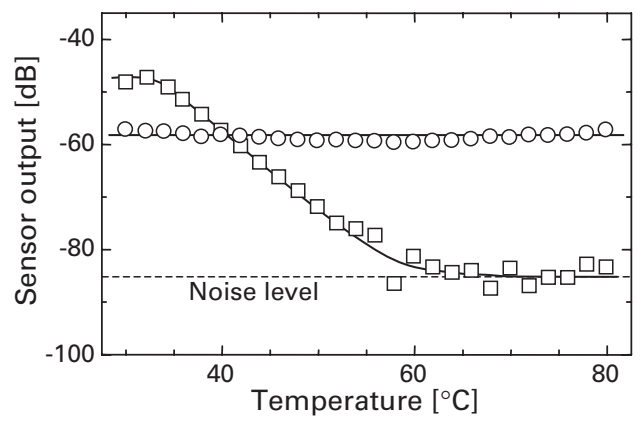

Fig. 8 Temperature dependence of sensor output: output with and without feedback control is plotted by open circle and open square, respectively.

It is noted that the output variation is estimated to be less than $2.5 \mathrm{~dB}$ with the feedback control but the output without feedback control varies considerably. The minimum sensor output of approximately $-85 \mathrm{~dB}$ corresponds to the noise level of the detected signal. The output variation of the sensor without the feedback control is estimated to be more than $40 \mathrm{~dB}$. This output variation is caused by deviation from the operation point of the wavelength of the laser: the reflection spectrum shifts to longer wavelength with the increasing temperature but the oscillation wavelength of the laser is fixed so that the sensitivity of the sensor output is varied. Especially for the flat region of the top of the reflection spectrum, the sensitivity almost vanishes.

In the experiment the temperature-stabilized TDM operation of the hydrophone array using the feedback control circuit has been successfully demonstrated. In the present stage the time response of the feedback 
system is $2.2 \mathrm{sec}$, which is limited mainly by the Peltier controller of the DFB laser used. As can be seen in Fig. 5, this effect appears in the transient responses of the sensor outputs when the arrayed hydrophones are switched. Since the feedback control enables us to realize the temperature stabilization, the time response is important factor for a practical application of the hydrophone array proposed. Though the temperature stabilization ranging from 30 to $80{ }^{\circ} \mathrm{C}$ has been demonstrated in Fig. 8, it is left for further study to improve the time response of the feedback control system including the laser source.

\section{Conclusion}

Combining the wavelength-controlling feedback circuit with tunable DFB laser, the TDM operation of the FBG hydrophone array has been realized. The interrogation technique of the FBG hydrophone is based on an intensity-modulation scheme, in that a tunable laser is used for an optical source of which oscillation wavelength tuned to a linear potion of a slope of the reflection spectrum curve of the FBG used. The partially reflected light from the FBG is modulated in intensity by an acoustic wave and offers a sensor output. For fabricating the FBG hydrophone array, an optical switch was used, in which the arrayed FBG hydrophones were connected in parallel with the output ports of the optical switch and the TDM operation was performed by changing the optical paths to the hydrophones. For the TDM operation, the feedback circuit was developed to control the oscillation wavelength automatically in accordance with the operation point that changes or shifts by exchange of FBG in the TDM operation or temperature variation. In the experiment, the operation principle of the feedback control circuit was confirmed and the thermally stabilized operation was demonstrated. For the thermal stabilization, the output variation without feedback control was estimated to be more than $40 \mathrm{~dB}$ whereas that with the feedback control was estimated to be less than $2.5 \mathrm{~dB}$. In our demonstration, two-point TDM underwater sound detection has been realized using twochannel optical switch, but a TDM operation for a large number of FBG hydrophones can be achieved using an optical switch with more than two channels.

\section{References}

1) B. S. Kawasaki, K. O. Hill, D. C. Johnson, and Y. Fujii, "Narrow-band Bragg reflectors in optical fibers," Opt. Lett., 3, 66-68 (1978).

2) K. O. Hill and G. Meltz, "Fiber Bragg grating technology fundamentals and overview," J. Lightwave Technol., 15, 1263-1276 (1997).

3) P. St. Russell and J. L. Archambault, "Fiber gratings," in Optical Fiber Sensors, Vol. 3, B. Culshaw and J. Dakin Eds. (Artech House, Boston, 1996) Chap. 2, pp. 9-67.

4) J. L. Santos and L. A. Ferreira, "Fiber Bragg grating interrogation techniques," in Handbook of Optical Fiber Sensing Technology, J. M. LpóezHiguera Ed. (John Wiley \& Sons, New York, 2002) Chap. 18, pp.379-402.

5) A. D. Kersey, M. A. Davis, H. J. Patrik, M. LeBlanc, K. P. Koo, C. G. Askins, M. A. Putnum, and E. J. Friebele "Fiber Bragg grating sensors," J. Lightwave Technol., 15 1442-1463 (1997).

6) N. Takahashi, K. Yoshimura, and S. Takahashi, "Detection of ultrasonic mechanical vibration of a solid using fiber Bragg grating," Jpn. J. Appl. Phys. Part 1, 39, 3134-3138 (2000).

7) N. Takahashi, K. Yoshimura, and S. Takahashi, "Fiber Bragg grating vibration sensor using incoherent light," Jpn. J. Appl. Phys. Part 1, 40, 36323636 (2000).

8) S. Tanaka, T. Ogawa, W. Thongnum, N. Takahashi, and S. Takahashi, "Thermally stabilized fiber-Bragg-grating vibration sensor using erbium-doped fiber laser," Jpn. J. Appl. Phys. Part 
1, 42, 3060-3062 (2003).

9) N. Takahashi, K. Tesumura, and S. Takahashi, "Underwater acoustic sensor using optical fiber Bragg grating as detecting element," Jpn. J. Appl. Phys. Part 1, 38, 3233-3236 (1999).

10) N. Takahashi, K. Yoshimura, S. Takahashi, and K. Imamura, "A compact optical fiber hydrophone using fiber Bragg grating," J. Mar. Acoust. Soc. Jpn., 27, 28-34 (2000).

11) P. Fomitchov and S. Krishnaswamy, "Response of a fiber Bragg grating ultrasonic sensor," Opt.
Eng., 42, 956-963 (2003).

12) N. Takahashi, T. Saeki, K. Tetsumura, S. Takahashi, and K. Imamura, "Pressure and temperature dependence of fiber Bragg grating for acoustic sensing," J. Mar. Acoust. Soc. 26, 231-238 (1999).

13) S. Tanaka, T. Ogawa, H. Yokosuka, and N. Takahashi, "Multiplexed fiber Bragg grating vibration sensor with temperature compensation using wavelength-switchable fiber laser," Jpn. J. Appl. Phys. Part 1, 43, 2969-2972 (2004). 